\title{
DISLOCATION AND FRACTURE-DISLOCATION OF THE HIP
}

\author{
R. A. JASKULKA, G. FISCHER, G. FENZL
}

\author{
From the Second Clinic of Trauma Surgery, University of Vienna
}

\begin{abstract}
Of 54 patients with posterior dislocations of the hip of type I and type II (Stewart and Milford 1954), 47 were followed for a mean period of 6.7 years $(2$ to 11). Of these, 23 had dislocation with minimal lesions of the acetabulum (type I) and 24 had an avulsed dorsocranial fragment (type II). All were reduced by closed methods within six hours. The subsequent treatment of type I dislocations was conservative. At the beginning of the period type II injuries were treated conservatively, but surgery was increasingly chosen for later cases.

Type I dislocations had significantly better results $(p<0.05)$ than type II fracture-dislocations, regardless of the method of treatment. There were no essential differences between the results of surgical and conservative treatment in type II dislocations.
\end{abstract}

There are differing views on the prognosis of simple dislocation of the hip: most authors report relatively few complications (Kristensen and Stougaard 1974; Reigstad 1980 ), but others have found arthritis in up to $40 \%$ (Brav 1962 ) and avascular necrosis in up to $6 \%$ (Hougaard and Thomsen 1987). The choice of treatment is also unclear. Operation is usual where there is extensive damage to the acetabulum or an associated fracture of the head or the neck of the femur, but for simple dislocation with no obvious damage to the acetabular rim recommendations range from traction with several weeks of bed-rest (Hougaard and Thomsen 1987) to early, full mobilisation (Stewart, McCarroll and Mulhollan 1975). Where there has been avulsion of a single, more or less large acetabular fragment, it has been suggested that surgical treatment offers no advantages over conservative therapy (Hougaard and Thomsen 1987).

We have reviewed the treatment and the late results in all our patients with hip dislocations treated from 1977 to 1987.

Classification. We used the system developed by Stewart and Milford (1954):

Type I, simple dislocation without fracture;

Type II, dislocation with avulsion of one or more fragments from the acetabular rim;

Type III, dislocation with destruction of the dorsal acetabulum and gross instability;

R. A. Jaskulka, MD, Assistant Professor

G. Fischer, MD, Chief Resident

G. Fenzl, MD, Resident

2nd Clinic of Trauma Surgery, University of Vienna, Spitalgasse 23, A-1090 Vienna, Austria.

Correspondence should be sent to Dr R. A. Jaskulka.

(C) 1991 British Editorial Society of Bone and Joint Surgery 0301-620X/91/3134\$2.00

J Bone Joint Surg [Br] 1991 ; 73-B:465-9.
Type $I V$, dislocation with fracture of the neck or head of the femur.

Type I dislocations are always stable after reduction, whereas type III dislocations are always unstable. Type II dislocations lie somewhere between the two, but the tendency is towards stability.

\section{PATIENTS AND METHODS}

From 1977 to 1987, a total of 54 adults with type I or type II posterior dislocation of the hip were treated at our clinic. We excluded patients with acetabular dysplasia. Five patients failed to attend for follow-up, one died of other injuries shortly after admission and a second patient died later. This left 47 patients for review at a mean of 6.7 years after injury (range 2 to 11 ). The age range at injury was 17 to 56 years (mean 29.7); there were 37 men and 10 women. The right hip was involved in 24 cases, and the left in 23 . Twenty-three patients had type I dislocations $(49 \%)$, with only minimal lesions of the acetabulum; 24 had type II with an avulsed dorsocranial fragment $(51 \%)$.

A road traffic accident was the cause of injury in $\mathbf{4 5}$ patients; 25 of whom were car drivers and 14 were passengers. Of these 39 , only 19 were wearing a seat-belt when the accident occurred. Six patients had been riding motorcycles or bicycles which were involved in collisions with cars. Two patients had fallen at work. Forty-five patients $(96 \%)$ had associated injuries, eight with severe polytrauma (Table I). Three patients $(6.4 \%)$ had primary lesions of the sciatic nerve.

Treatment.

We regard every hip dislocation as an emergency until it has been reduced. This, combined with the taking of routine pelvic radiographs when an injured patient is drowsy or comatose, has insured that all dislocations are 
R. A. JASKULKA, G. FISCHER, G. FENZL

reduced by closed methods within six hours of the injury. In all cases, reduction was under general anaesthesia using traction on the flexed leg in the supine position. In patients with associated injuries of the lower leg or knee, traction was applied by means of a Steinmann pin inserted above the femoral condyles.

Table I. Associated injuries in 54 patients with type I and type II dislocations of the hip

\begin{tabular}{lc}
\hline & Number \\
\hline Head injury & 28 \\
Acromioclavicular subluxation & 2 \\
Shoulder dislocation & 2 \\
Fracture of the humerus & 2 \\
Fracture of the forearm or hand & 12 \\
Thoracic injury & 3 \\
Spinal fracture & 2 \\
Femoral shaft fracture & 6 \\
Tibial fracture & 4 \\
Knee ligament injury & 7 \\
Below-knee amputation & 1 \\
Metatarsal fracture & 9 \\
\hline
\end{tabular}

was gradually replaced by an increasing tendency towards surgical treatment. The main reason for this was the improved accuracy of diagnosis achieved with CT scans. Conservative treatment was also used in cases where there were local or general contra-indications to surgery.

Conservative treatment in this group consisted of an average of three weeks traction after reduction. Partial weight-bearing was permitted on average six weeks after injury, and full weight-bearing after an average of 12 weeks.

Operation was performed in all cases requiring acetabular reconstruction or the removal of intraarticular fragments. Following primary reduction and traction, surgery took place after an average of 14.3 days. A posterior approach was used in all cases to avoid further devascularisation of the femoral head, special attention being given to the visualisation and protection of the sciatic nerve. After precise reduction of the avulsed fragment, stabilisation was effected with compression screws.

After operation, no traction was applied, and no weight-bearing was allowed for six weeks. Full weightbearing was allowed from 12 weeks.

Review. At follow-up, patients were asked about their symptoms and then given a thorough clinical examination, with tests of hip function and range of movement compared with the contralateral hip. Radiographs in two planes were compared with the initial films and those taken during follow-up.

Table II. Criteria used to evaluate results after hip dislocation (Epstein 1974)

\begin{tabular}{lllll}
\hline Grade & Pain & Limp & $\begin{array}{l}\text { Range of } \\
\text { movement }\end{array}$ & Radiograph \\
\hline Excellent & None & None & Full & $\begin{array}{l}\text { No progressive changes } \\
\text { Good }\end{array}$ \\
Slight & None & $>75 \%$ & $\begin{array}{l}\text { Minimal changes } \\
\text { No avascular necrosis or } \\
\text { narrowing }\end{array}$ \\
Fair & $\begin{array}{l}\text { Mild to } \\
\text { moderate }\end{array}$ & Slight & $50 \%$ to $75 \%$ & $\begin{array}{l}\text { Moderate changes } \\
\text { Narrowing, cyst formation } \\
\text { Acetabular sclerosis }\end{array}$ \\
Poor & Severe & Definite & $\begin{array}{l}\text { Marked } \\
\text { limitation }\end{array}$ & Progressive degeneration \\
\hline
\end{tabular}

Further treatment varied according to the type of dislocation. In type I dislocations without an acetabular lesion, patients had bed-rest, with no weight-bearing for three weeks. Full weight-bearing was started an average of six weeks after injury ( 10 days to 7 weeks). No patient was fully immobilised; during bed-rest, all had physiotherapy with passive joint movements.

For type II dislocations with avulsion of a dorsocranial fragment, conservative management after reduction
Patients were then grouped as simple dislocations (type $\mathrm{I}, \mathrm{n}=23$ ), and those with a significant avulsion fracture (type II, $n=24$ ). The latter were further classified according to the treatment given: conservatively treated cases (type $I I /$ cons, $n=10$ ), were distinguished from those treated surgically (type II/op, $\mathrm{n}=14$ ).

The individual results within each group were compared and averaged for the group. For comparison 
with other series, the results were also classified according to Epstein's (1974) version of Stewart and Milford's classification (1954). This system of radiological and clinical parameters is in general use (Table II). The scores were then subjected to further statistical evaluation.

\section{RESULTS}

Pain. Three patients complained of constant pain (Table III). Two with type I injuries were active sportsmen, and the third (type II/op) had had a total hip replacement for avascular necrosis - the only case in our series.

Ability to work. No patient was unable to work because of the hip injury. However, associated injuries had forced about half of the patients to change their occupation. One patient receives a $100 \%$ disability pension after above-knee amputation of one leg and below-knee amputation of the other.

Gait. Two patients, including a bilateral amputee and a patient who had hip replacement, had severe limps. In addition, two patients with residual peroneal paresis had slight limps, but no patient had an abnormal gait as a direct consequence of hip dislocation. Subjective opinions on walking ability and the measured range of hip movement are also given in Table III.

Neurological status. Two patients with primary nerve lesions still had minimal peroneal weakness after conservative treatment. A third patient, treated surgically, had no residual neurological symptoms. One patient with postoperative sciatic weakness continued to have im-

Table IV. Overall results in the three treatment groups (see also Figure 1)

\begin{tabular}{llrll}
\hline & & \multicolumn{3}{l}{ Type II } \\
\cline { 4 - 5 } \cline { 4 - 4 } Objective & Score & Type I & Conservative & Operative \\
\hline & Excellent & 14 & 2 & 2 \\
& Good & 3 & 2 & 6 \\
& Fair & 5 & 6 & 5 \\
Subjective & Poor & 1 & 0 & 1 \\
& Excellent & 16 & 8 & 6 \\
& Good & 0 & 0 & 0 \\
& Fair & 6 & 2 & 7 \\
& Poor & 1 & 0 & 1 \\
\hline
\end{tabular}

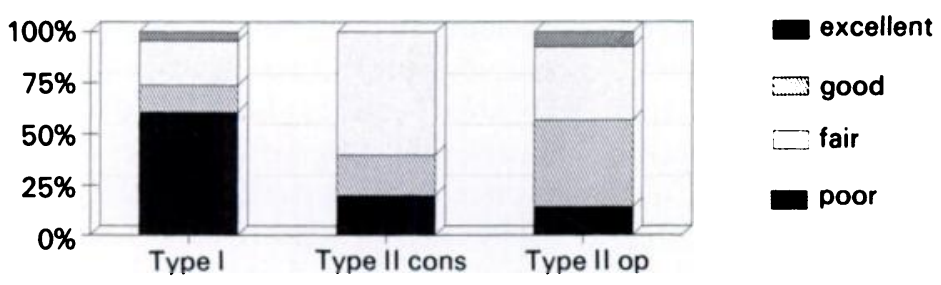

Fig. 1a

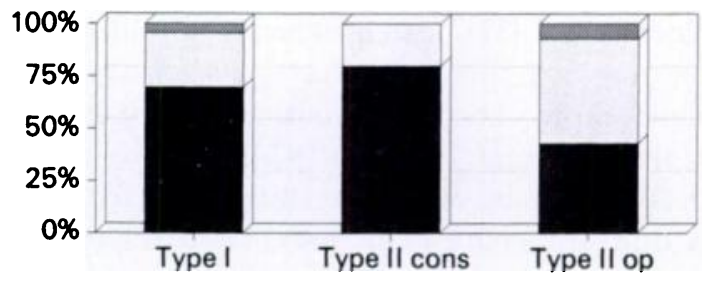

Fig. 1b

The results of treatment by percentage (see Table IV). Figure la - Objective assessment. Figure lb - Patients'opinions.

Table III. Detailed results in 54 patients with dislocation of the hip in three treatment groups (see text)

\begin{tabular}{llrll}
\hline & & & \multicolumn{2}{l}{ Type II } \\
\cline { 3 - 5 } & Score & Type I & Conservative & Operative \\
\hline Pain & Excellent & 18 & 4 & 8 \\
& Good & 3 & 6 & 5 \\
& Fair & 0 & 0 & 0 \\
& Poor & 2 & 0 & 1 \\
Walking & Excellent & 16 & 8 & 7 \\
& Good & 7 & 2 & 6 \\
& $>1$ km & 0 & 0 & 0 \\
& $<1$ km & 0 & 0 & 1 \\
Range of movement & Excellent & 12 & 8 & 4 \\
& Good & 6 & 2 & 8 \\
& Lost $<20^{\circ}$ & 5 & 0 & 1 \\
& Lost $>20^{\circ}$ & 0 & 0 & 1 \\
Radiograph & & & & \\
& Excellent & 20 & 4 & 7 \\
& Good & 2 & 4 & 6 \\
& Fair & 0 & 2 & 0 \\
& Poor & 1 & 0 & 1 \\
\hline
\end{tabular}

paired sensation over the lateral side of the lower leg but no motor impairment.

Radiography. Compared with the contralateral hip, two patients had fair results (Table III). Two had poor results, one who had had total hip replacement and the other who had severe osteoarthritis.

The overall objective results are given in Figure la and Table IV. Of the 23 type I dislocations, $61 \%$ had excellent results. Of the 10 type II/con, $20 \%$ were excellent, $20 \%$ were good, $60 \%$ fair, and there were no poor results. Of 14 type II/op, $14 \%$ were excellent, $35 \%$ fair, and $7 \%$ poor. The difference between the results for type I and type II (cons plus op) was significant at $p<0.05$. There was no significant difference between the results of type II/cons and type II/op.

The subjective results are given in Figure $1 \mathrm{~b}$ and Table IV. There was only partial correlation between subjective and objective findings. In all groups, and 
particularly type II dislocations, the patients' own evaluation was the better. However, the two patients who were very dissatisfied also had poor objective results.

\section{DISCUSSION}

Traumatic dislocation of the hip usually results from an impact of considerable force: $96 \%$ of our series had traffic accidents, and it is worth noting that less than half the patients injured in a vehicle were wearing seat belts. This confirms the views of other authors (Epstein 1974; Stewart et al 1975; Rosenthal and Coker 1979) that the use of seat belts can often prevent this type of injury.

Many patients presented with severe concomitant injuries, so no uniform treatment was possible, particularly as regards mobilisation and duration of non-weightbearing, even for simple dislocations. However, such associated injuries were evenly distributed between the two types of dislocation, confirming the generally accepted view that the degree of external force does not influence the type of dislocation (Upadhyay, Moulton and Burwell 1985).

In the literature, there are a relatively large number of cases with late reductions due to late diagnosis (Epstein 1974; Kristensen and Stougaard 1974; Hougaard and Thomsen 1987), often when the patient had been unconscious. It has therefore been advocated that pelvic radiography should be a routine investigation in cases of multiple trauma (Epstein 1974). We have done this: all dislocations in our series were detected and reduced within six hours. The prognostic importance of the earliest possible diagnosis and reduction, particularly in relation to avascular necrosis, is widely recognised (Stewart and Milford 1954; Epstein 1974; Stewart et al 1975; Rosenthal and Coker 1979; Reigstad 1980; Hougaard and Thomson 1986, 1987), and the fact that we had only one case of avascular necrosis seems to confirm this view.

It has been reported that up to $4 \%$ of simple dislocations cannot be reduced conservatively (Reigstad 1980; Upadhyay and Moulton 1981; Hougaard and Thomsen 1987), but we had no such experience. However, we agree that if there is an obstacle to closed reduction, then primary surgical management is indicated (Epstein 1974).

Our management of type I dislocations included three weeks bed-rest and an average of six weeks nonweight-bearing. We agree with others that in these cases (which are always stable after reduction) traction is unnecessary, even with regard to the risk of avascular necrosis. This is confirmed by our long-term results after simple dislocation: $87 \%$ were free from osteoarthritis, none had avascular necrosis, and $74 \%$ were graded good or excellent. Our findings are comparable with those obtained by other authors who recommend a maximum period of six weeks non-weight-bearing (Kristensen and Stougaard 1974; Stewart et al 1975; Hougaard and
Thomsen 1987). Our results were no worse than those after a longer period of non-weight-bearing (Epstein 1974). One patient from this group did, however, have an extremely poor radiographic result of uncertain cause. There were definite degenerative changes and limitation of joint movement, but despite these findings, the patient reported almost complete freedom from symptoms.

The results after type I dislocations were significantly better $(p<0.05)$ than after type II dislocations, regardless of the method of treatment. This confirms the experience of others (Upadhyay and Moulton 1981; Hougaard and Thomsen 1986) who found that the primary extent of injury has a significant effect on prognosis.

We treated type II injuries conservatively at the beginning of the series, but later on increasingly chose operation, with careful attention to precise reduction and functionally stable osteosynthesis. However, despite this, we found no essential difference in the conservative and surgical treatment. In terms of subjective evaluation and measurable joint function, conservatively treated patients had better results, though no statistical significance could be established. Similar findings have been reported (Brav 1962; Rosenthal and Coker 1979; Hougaard and Thomsen 1987).

We therefore consider that the technically complicated and time-consuming surgical stabilisation of fragments in type II dislocations can only be justified when a definite positive influence on prognosis can be shown, when for example there is persistent instability. By definition, this is rarely seen in type II dislocations as against type III; the avulsion of an acetabular fragment should not, on its own, be an indication for surgery.

In our series, the conservative management of type II dislocations was traction for three weeks. However, when the joint is stable, this may not improve the prognosis (Hougaard and Thomsen 1986). We consider that it should be used only for those cases with evidence of instability where there are local or general contraindications to surgery. Stable injuries, on the other hand, are probably best managed without immobilisation and with the addition of regular exercise on a passive motion device.

Intra-articular cartilage or bone fragments are always an indication for surgery; we do not agree with those who consider that fragments outside the immediate weight-bearing zone are unimportant (Rosenthal and Coker 1979). The detection of such fragments is helped by tomography and more recently by CT scanning (Ordway and Xeller 1984). In the last few years we have used CT scans more regularly in patients with type II dislocations; this is more useful in practice than conventional tomography, especially if there is any doubt about the size or the location of the fragment. CT scans can also provide valuable information about the integrity of the joint space and fractures of the femoral head. Neurological symptoms represent a further indication 
for operation, above all where the cause may be an avulsed acetabular fragment. Although neurological symptoms generally tend to improve, full recovery is, in many cases, only possible after reduction of the fragment. Our results confirm this supposition; in one case, full neurological recovery followed surgical treatment, while two patients who had been treated conservatively have residual weakness of the peroneal musculature.

\section{Conclusions}

1) Routine radiography of the pelvis is essential in cases of multiple trauma, especially in cases with head injury.

2) Every dislocated hip should be reduced as soon as possible, preferably in the emergency room.

3) The end results of type II dislocations are inevitably poorer than those of type I, regardless of the method of treatment.

4) A relatively short period of non-weight-bearing is sufficient for type I dislocations. Full weight-bearing is safe after six weeks. Traction is no longer considered to be the treatment of choice.

5) If there is any doubt as to the size or location of a fragment, a CT scan is advisable to help decide if an operation is necessary and to help plan the surgical approach.

6) Surgical reduction of an avulsed fragment of the acetabular rim is only necessary in cases of persistent instability after initial reduction. However, if there is a primary neurological injury, particularly in type II injuries, the sciatic nerve should be visualised and the fragment reduced.
7) In type II dislocations, traction is necessary only if surgery is contra-indicated for local or general reasons.

No benefits in any form have been received or will be received from a commercial party related directly or indirectly to the subject of this article.

\section{REFERENCES}

Brav EA. Traumatic dislocation of the hip: army experience and results over a twelve-year period. J Bone Joint Surg [Am] 1962; 44A:1115-34.

Epstein HC. Posterior fracture dislocations of the hip. J Bone Joint Surg [Am] 1974; 56-A :1103-27.

Hougaard K, Thomsen PB. Traumatic posterior dislocation of the hip prognostic factors influencing the incidence of avascular necrosis of the femoral head. Arch Orthop Trauma Surg 1986; $106: 32-5$.

Hougaard K, Thomsen PB. Coxarthrosis following traumatic posterior dislocation of the hip. J Bone Joint Surg [Am] 1987; 69-A :679-83.

Kristemsen O, Stougaard J. Traumatic dislocation of the hip: results of conservative treatment. Acta Orthop Scand 1974; 45:206-12.

Ordway CB, Xeller CF. Transverse computerized axial tomography of patients with posterior dislocation of the hip. J Trauma 1984; 24:76-9.

Reigstad A. Traumatic dislocation of the hip. J Trauma 1980; 20:603-6.

Rosenthal RE, Coker WL. Posterior fracture-dislocation of the hip: an epidemiologic review. J Trauma 1979; 19:572-81.

Stewart MJ, McCarroll HR, Mulhollan JS. Fracture-dislocation of the hip. Acta Orthop Scand 1975; 46:507-25.

Stewart MJ, Milford LW. Fracture-dislocation of the hip: an end result study. J Bone Joint Surg [Am] 1954; 36-A :315-42.

Upadhyay SS, Moulton A. The long-term results of traumatic posterior dislocation of the hip. J Bone Joint Surg [Br] 1981 ; 63-B:548-51.

Upadhyay SS, Moulton A, Burwell RG. Biological factors predisposing to traumatic posterior dislocation of the hip: a selection process in the mechanism of injury. J Bone Joint Surg [ Br] 1985; 67-B :232-6. 